\title{
Immortalized N/TERT keratinocytes: An excellent and versatile alternative for primary keratinocytes in experimental dermatological research
}

Jos P.H. Smits' ${ }^{1}$, Hanna Niehues ${ }^{1}$, Gijs Rikken ${ }^{1}$, Ivonne M.J.J. van Vlijmen-Willems ${ }^{1}$, Guillaume W.H.J.F.L. van de Zande², Patrick L.J.M. Zeeuwen', Joost Schalkwijk', Ellen H. van den Bogaard ${ }^{1}$

'Department of Dermatology, Radboud Institute for Molecular Life Sciences (RIMLS), Radboudumc, Nijmegen, The Netherlands ${ }^{2}$ Department of Human Genetics, Radboud University Medical Center (Radboudumc), Nijmegen, The Netherlands

\section{Introduction}

- In vitro 3D human epidermal equivalents (HEEs) are generally made using adult primary keratinocytes to mimick native skin.

- Disadvantages of human primary keratinocytes are - limited availability.

- short lifespan.

- inter-donor variation.

- Only few immortalized keratinocyte cell lines are available.

- The widely used HaCaT cell line shows aneuploidy, abnormal epidermal differentiation, abberant epidermal stratification and is less suitable for developing 3D skin models.

\section{Objective:}

To illustrate the potential of human keratinocyte cell lines (N/TERT1 and N/TERT2G) for studies on epidermal biology, inflammatory skin disease pathogenesis and therapeutics.

\section{Results}

N/TERT keratinocyte gene expression corresponds to that of human primary keratinocytes in monolayer cultures:
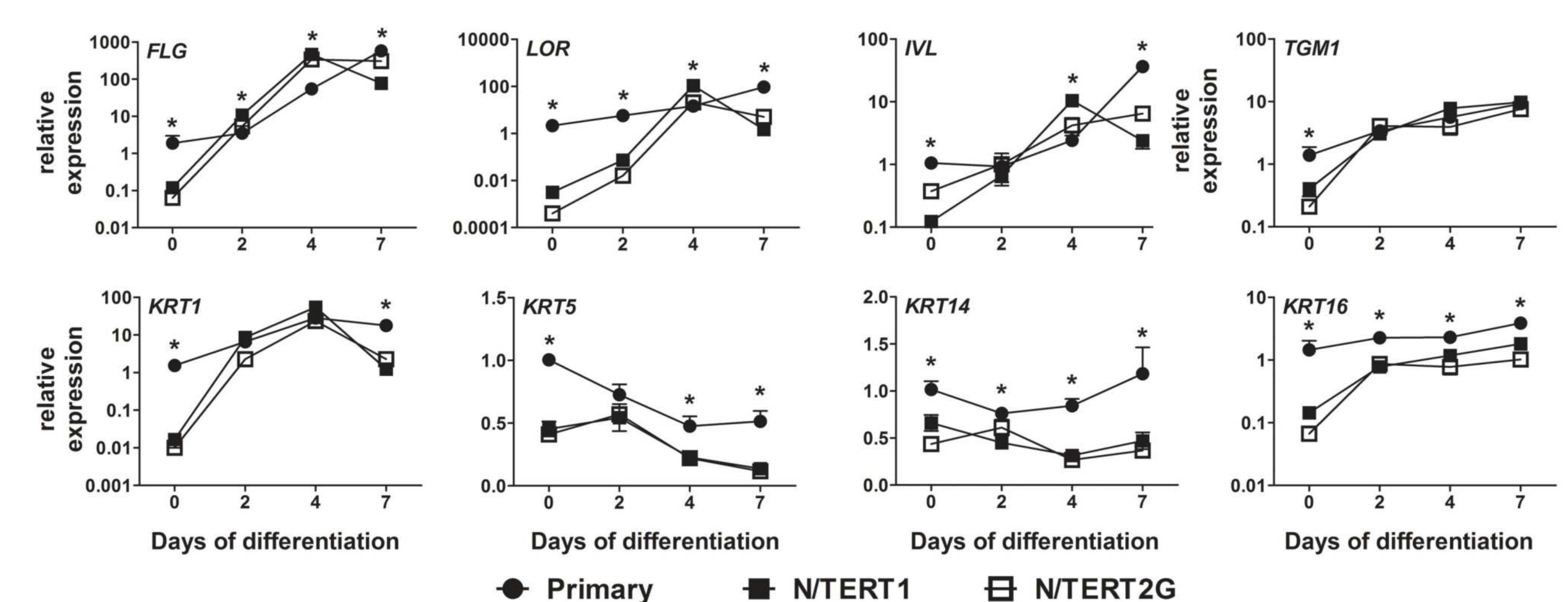

N/TERT keratinocyte cell lines are diploid:

N/TERT1 karyotype: $47, \mathrm{XY},+20$

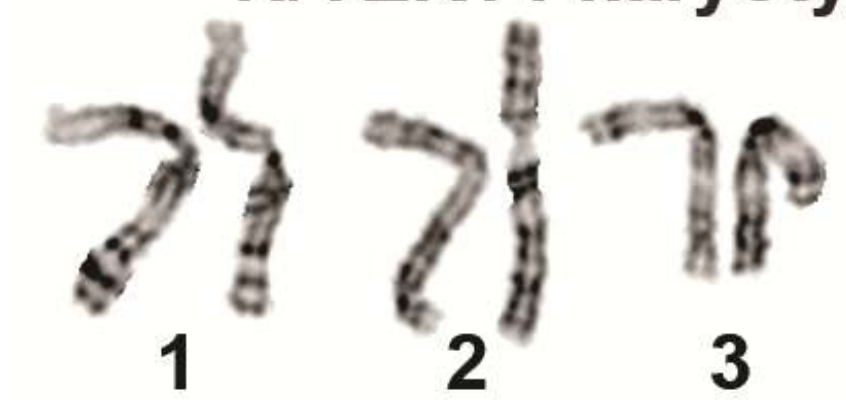

7 期

1f

औ It at at 10

$\begin{array}{lllll}14 & 15 & 16 & 17 & 18\end{array}$

88183

$19 \quad 20$

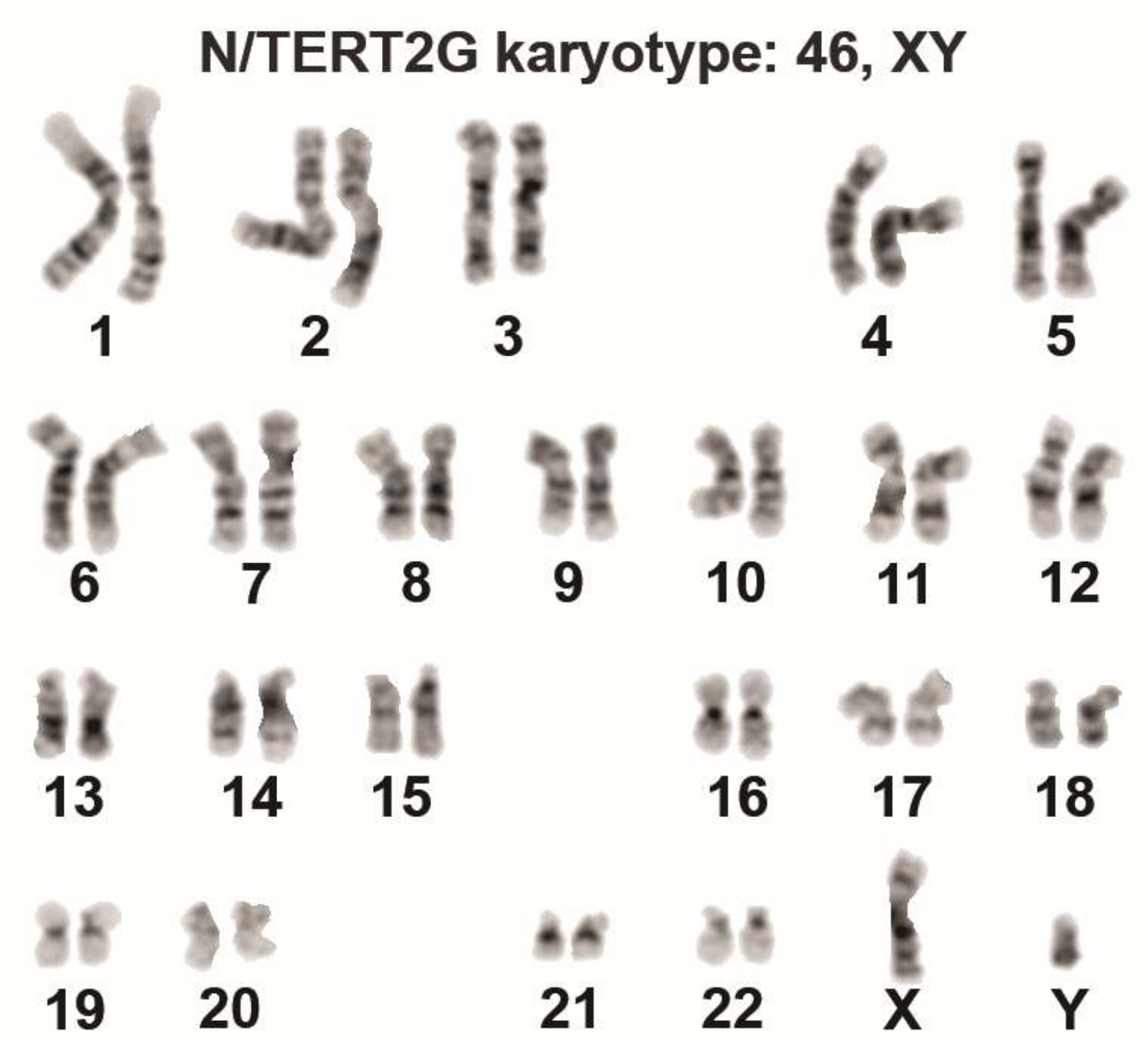

\section{Results}

High quality HEEs are generated from N/TERT keratinocytes:

$H \& E$
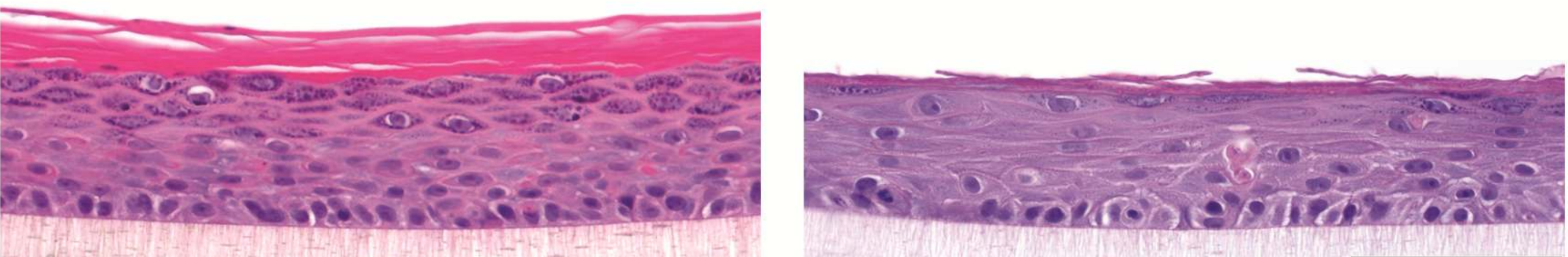

FLG
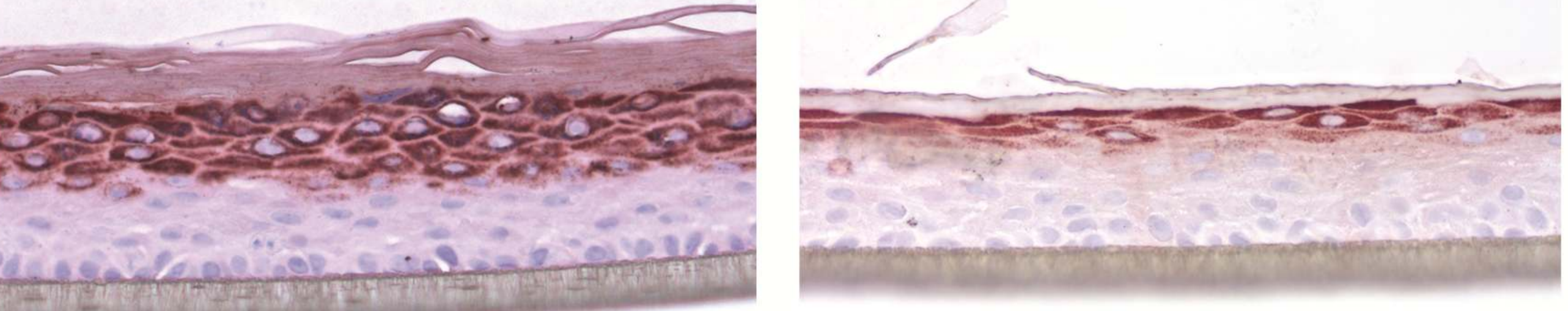

Ki-67
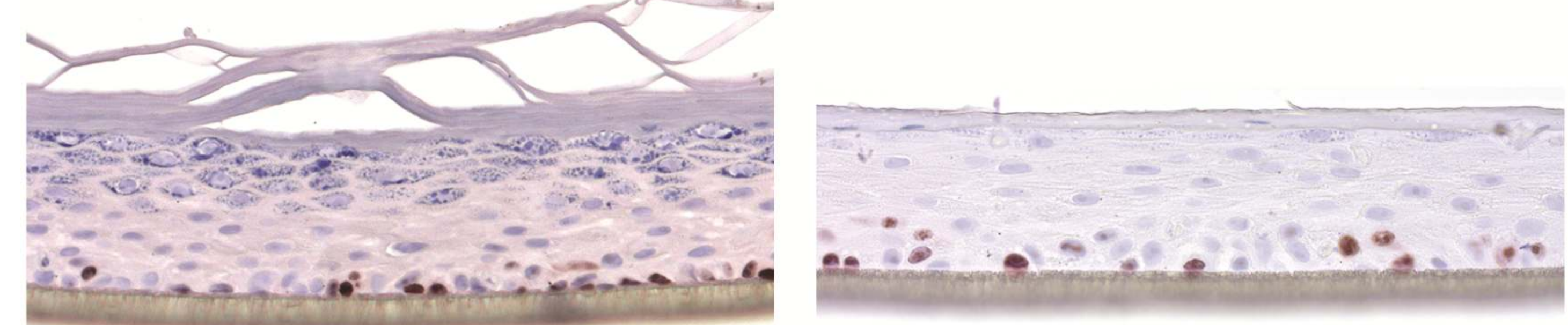

SKALP

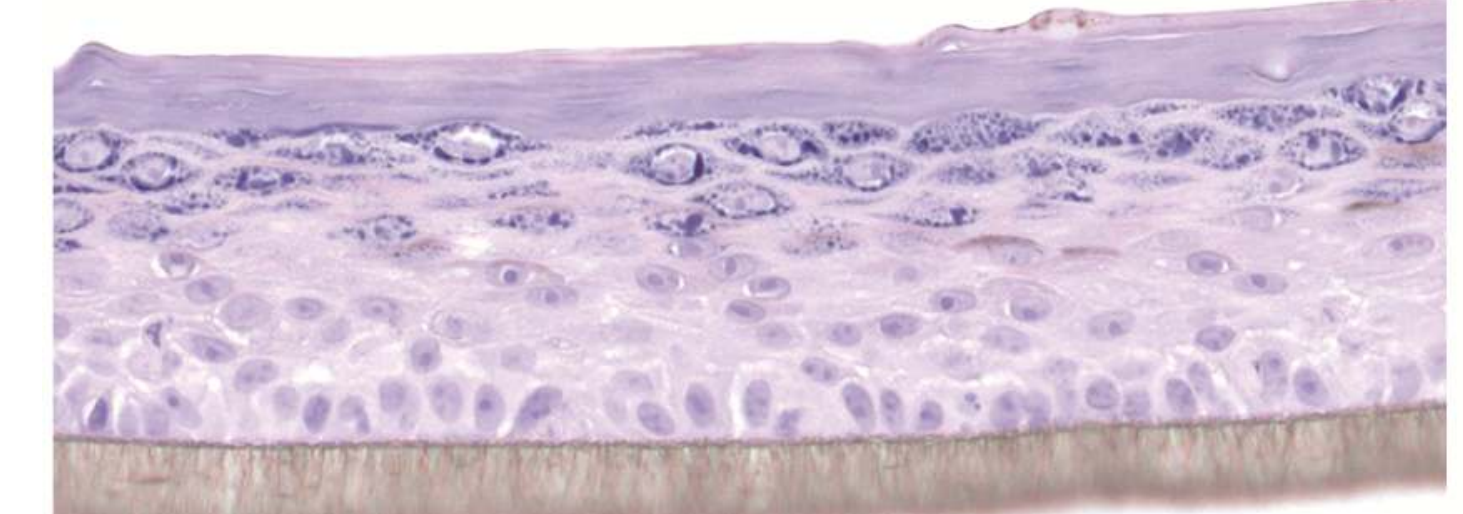

Primary keratinocytes

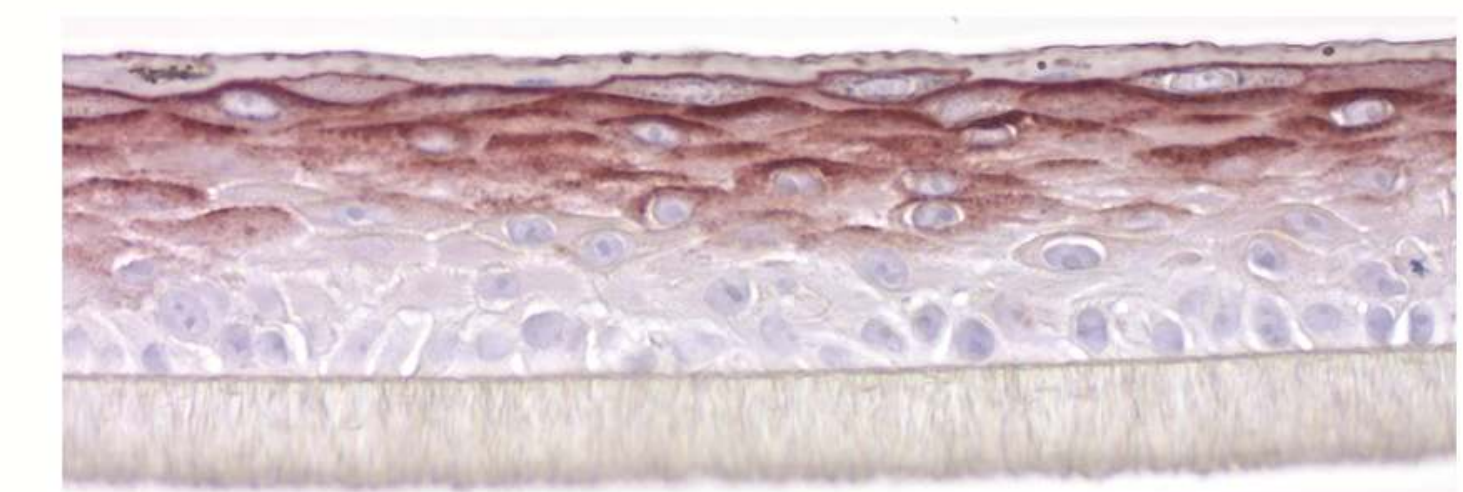

N/TERT keratinocytes

HEEs from N/TERT keratinocytes form a functional barrier:
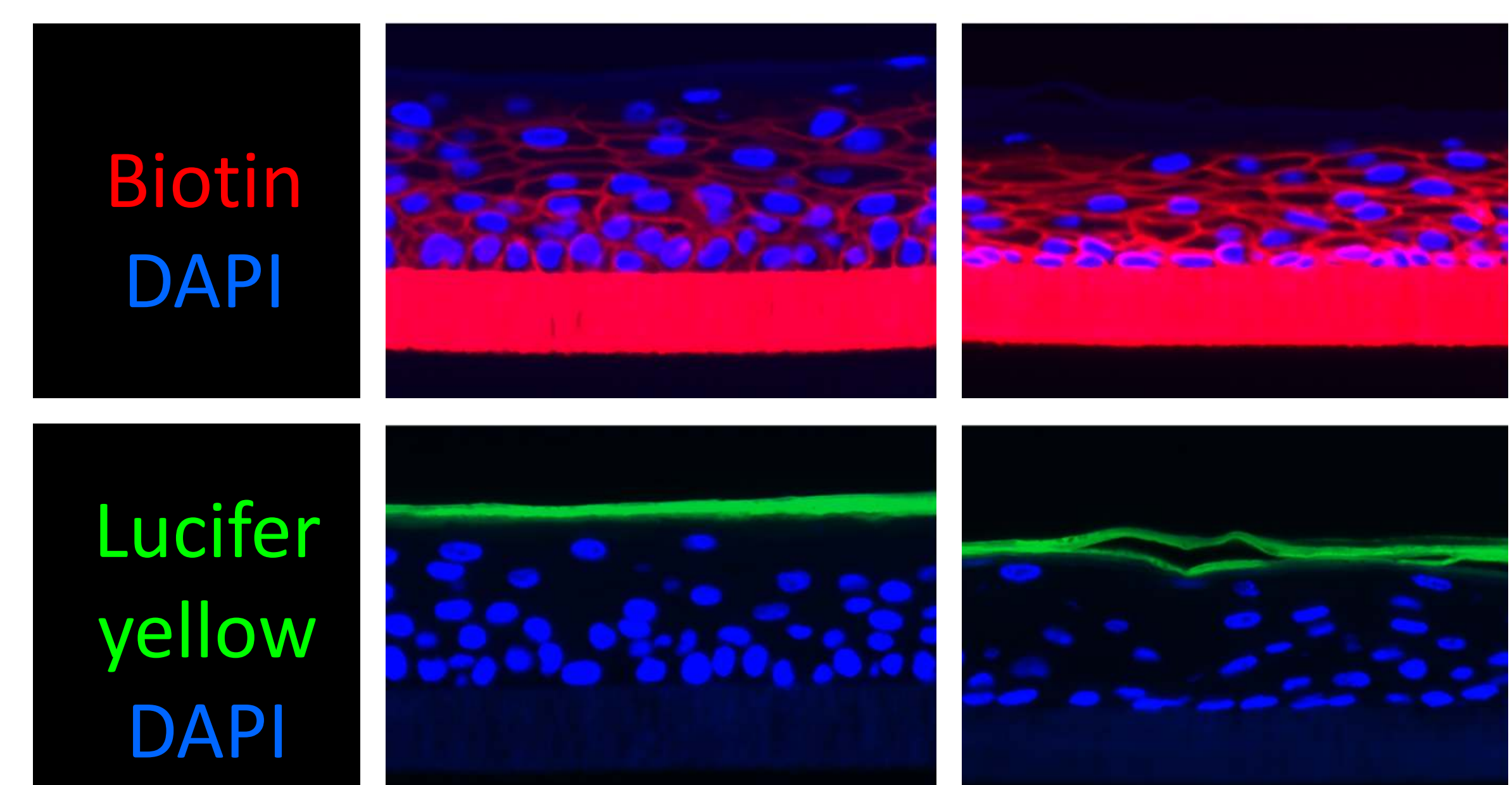

Day 6

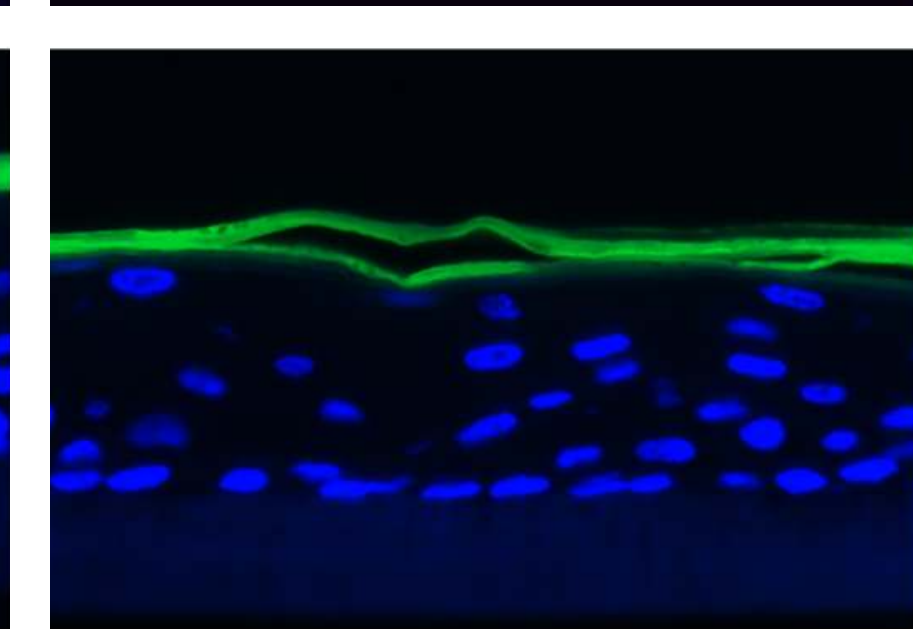

Day 8

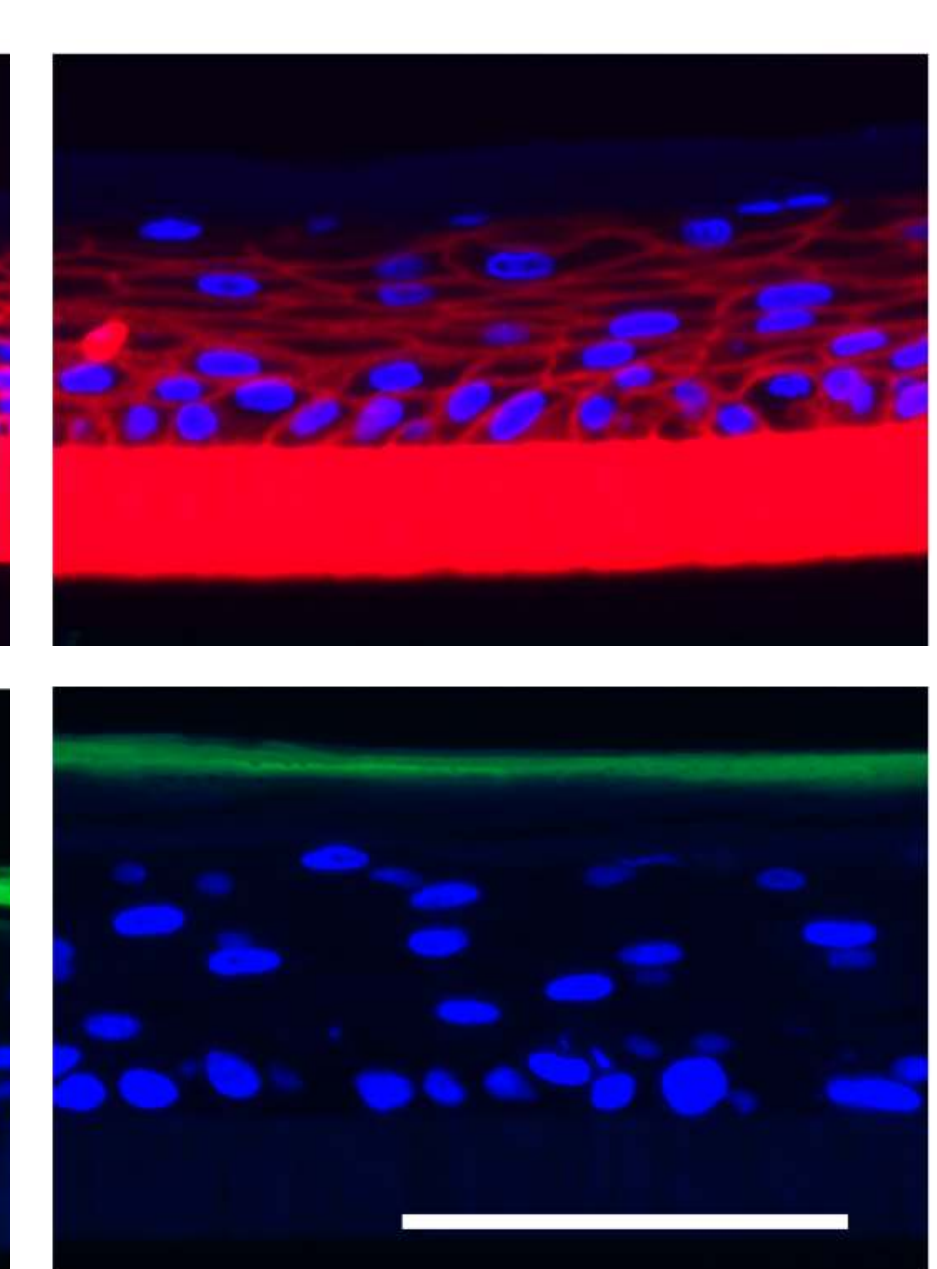

Day 10

Atopic Dermatitis (AD) hallmarks were induced in HEEs by Th2 cytokines and subsequently rescued by drug treatment:

$H \& E$
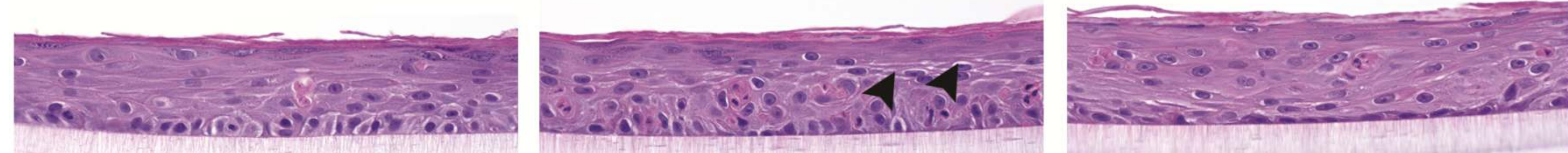

FLG
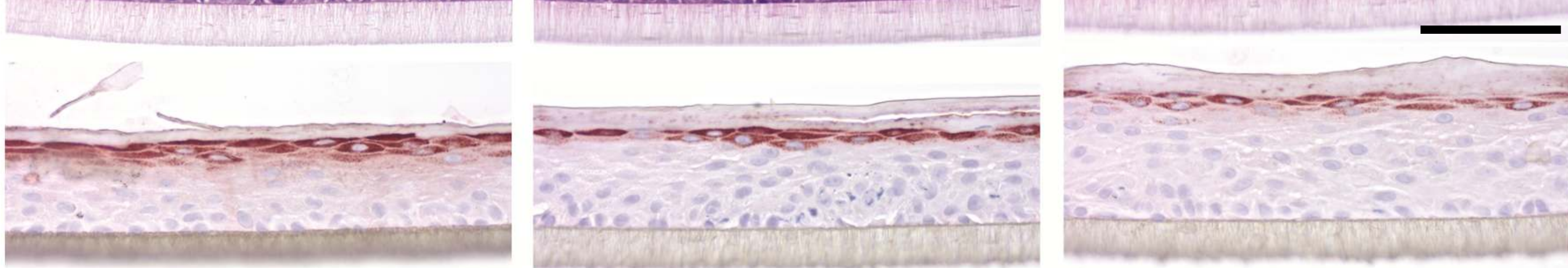

IVL
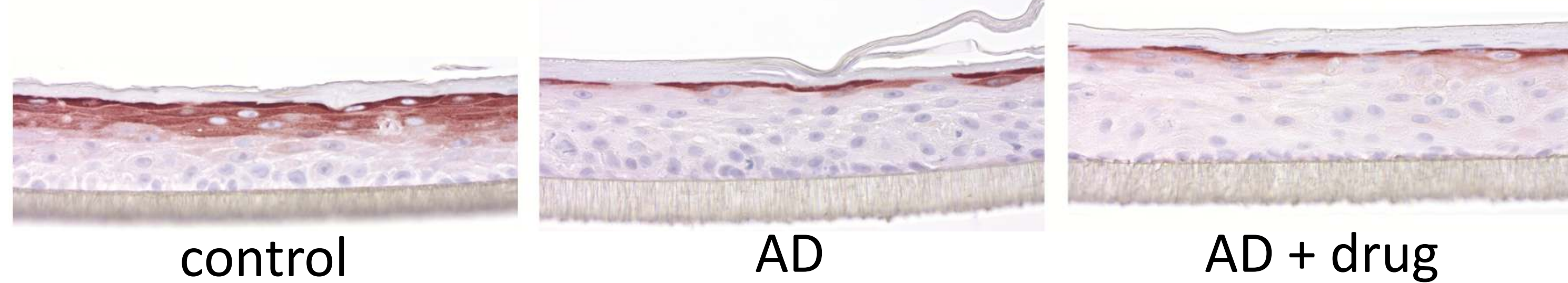

\section{N/TERT keratinocytes...}

...show high quality HEE morphology. Gene and protein expression is highly comparable to that of human primary keratinocytes. ...HEEs are useful to study epidermal biology, inflammatory skin disease pathogenesis, and therapeutics.

...have a great potential for implementation of advanced techniques (e.g. CRISPR-Cas9). 